\title{
Solução do átomo de hidrogênio usando Supersimetria
}

Solution of the hydrogen atom using Supersymmetry

\author{
João Marcos Costa Monteiro*1], Airton Algozini Júnior ${ }^{1}$, Elso Drigo Filho ${ }^{1}$ \\ ${ }^{1}$ Universidade Estadual Paulista, Instituto de Biociências, Letras e Ciências Exatas, São José do Rio Preto, SP, Brasil
}

Recebido em 13 de Novembro, 2018. Revisado em 12 de Março, 2019. Aceito em 07 de Maio, 2019.

\begin{abstract}
Nesse trabalho apresentamos a solução da equação de Schrödinger através de uma versão moderna do método de fatorização usando o formalismo da chamada Mecânica Quântica Supersimétrica. O sistema tratado é o átomo de hidrogênio e tanto a parte radial como a angular das coordenadas esféricas são tratadas com a metodologia apresentada. Especial destaque é dado às adequações necessárias a cada uma dessas coordenadas. A abordagem utilizada é geral e pode ser apresentada como alternativa aos métodos tradicionais de solução da equação de Schrödinger como, por exemplo, o método de Frobenius.

Palavras-chave: átomo de hidrogênio, supersimetria, shape invariance, operadores-escada generalizados, mecânica quântica.
\end{abstract}

In this work we present the solution of the Schrödinger equation through a modern version of the factorization method by using the formalism of the so-called Supersymmetric Quantum Mechanics. The analyzed system is the hydrogen atom, the radial and the angular parts of the spherical coordinates are studied with the presented methodology. Special emphasis is given to adaptation made to each of these coordinates. The approach used is general and can be presented as an alternative to traditional methods of solution of the Schrödinger equation such as the Frobenius method.

Keywords: hydrogen atom, supersymmetry, shape invariance, ladder operators, quantum mechanics.

\section{Introdução}

Devido a seu lugar de destaque no contexto da Mecânica Quântica, a equação de Schrödinger independente do tempo tem sido largamente estudada em seu aspecto Físico e Matemático. Textos iniciais de Mecânica Quântica [1-3] apresentam a solução dessa equação em coordenadas esféricas, para potenciais de força central. A solução indicada inclui a parte angular, dada em termos dos Harmônicos Esféricos, enquanto a parte radial é usualmente resolvida usando o Método de Froubenius. Grosso modo, na obtenção da solução radial, são explorados os limites assintóticos e com a variável tendendo a zero, o restante da solução é determinada por uma expansão em série de potências. Essa abordagem está tão difundida que por vezes há dificuldades de reconhecer que outros tratamentos podem ser usados para determinar os autovalores e autofunções da equação de Schrödinger.

O presente artigo tem como objetivo trabalhar com um método de obtenção da solução da equação de Schrödinger por uma metodologia distinta daquela explorada tradicionalmente em livros e textos didáticos. A abordagem seguida aqui envolve a fatorização das equações diferenciais e construção das autofunções através da generalização dos operadores escada. A versão moderna da metodologia empregada se enquadra no contexto da

*Endereço de correspondência: cmonteiro.jm@gmail.com
Mecânica Quântica Supersimétrica (MQS) [4-6]. A abordagem tratada nesse texto se restringe a potenciais com soluções exatas/analíticas, embora extensões para outros sistemas através de métodos aproximativos podem ser utilizadas. Exemplos dessas abordagens são a aproximação WKB $[7,8]$ e método variacional [4,9-12].

O sistema físico estudado nesse texto é o átomo de hidrogênio, ou seja, uma partícula sujeita a um potencial de força central proporcional a $-1 / r$. Como usual, esse problema é tratado em coordenadas esféricas. Com a abordagem usando a superálgebra, normalmente, apenas a parte radial dessa equação é analisada. Isso é compreensível, uma vez que os autovalores de energia emergem da equação radial. Assim, vários trabalhos que se propõem a tratar o átomo de hidrogênio usando o formalismo de fatorização têm se restringido a trabalhar apenas com a parte dependente da variável $r$ [3,4,13]. Também há trabalhos que calculam a função de onda azimutal (correspondente à variável angular $\theta$ ) [14,15]. Entretanto, para a variável angular polar $(\varphi)$, até onde vai o nosso conhecimento, nada foi escrito usando de forma direta o formalismo da MQS. Neste caso, é necessária uma adaptação no tratamento para incorporar, não apenas a solução da equação diferencial, mas também a condição de contorno física apropriada ao problema.

A metodologia seguida aqui implica que o Hamiltoniano usado para descrever o sistema seja fatorizado por um par de operadores supersimétricos. A inversão 
da ordem desses operadores permite identificar o Hamiltoniano companheiro supersimétrico do original. A exploração dessa propriedade leva à construção de uma cadeia de Hamiltonianos relacionados entre si através dos operadores supersimétricos que é conhecida como hierarquia de Hamiltonianos [4-6].

O caminho seguido aqui é apropriado para potenciais quânticos que são invariantes na sua forma funcional por transformações de supersimetria (shape invariance) [4,16-23]. Para esses potenciais, é possível obter a solução do problema através da construção de operadores de criação e destruição que atuam simultaneamente na coordenada espacial e no parâmetro relacionado à shape invariance. Uma vantagem da construção desses operadores escada é que eles permitem determinar estados coerentes para os sistemas analisados [24-27].

Em termos de organização desse artigo, na seção 2 a metologia adotada é apresentada. Na seção 3, o Hamiltoniano do átomo de hidrogênio é escrito em coordenadas esféricas e é indicada a separação da equação diferencial original nas três equações, uma para cada coordenada espacial. Nas seções 4, 5 e 6 são tratadas de forma individual as equações para cada uma das coordenadas, $\varphi, \theta$ e $r$, respectivamente. Por fim, são apresentadas e discutidas as soluções gerais do problema e apresentadas as conclusões do trabalho.

\section{Metodologia}

Uma revisão recente do formalismo matemático utilizado nesse trabalho pode ser encontrado no artigo de Batael et all [28]. Entretanto, apresentamos os pontos principais da metodologia para tornar esse texto mais compreensível e didático.

O passo inicial para aplicação do formalismo utilizado é identificar o Hamiltoniano que emerge da equação de Schrödinger para um potencial $V(x)$, usado para descrever o sistema sob estudo. De forma geral, esse Hamiltoniano pode ser escrito como:

$$
H=-\frac{d^{2}}{d x^{2}}+V(x)
$$

Note que não há restrição sobre a natureza da coordenada $x$, ela pode ser cartesiana ou não. Foi adotado, por simplicidade, $\hbar=2 \mu=1$, sendo que $\hbar$ é a constante de Planck e $\mu$ é a massa reduzida do sistema. Essa simplificação não altera a apresentação dos conceitos, mas ajuda a não sobrecarregar a notação.

Os operadores bosônicos, $\mathrm{A}^{+}$e $A^{-}$, são definidos pelas expressões:

$$
\begin{aligned}
& A^{+}=-\frac{d}{d x}+W(x ; a) \\
& A^{-}=\frac{d}{d x}+W(x ; a),
\end{aligned}
$$

onde $W(x ; a)$ é chamado de superpotencial. É importante notar que os operadores $A^{ \pm}$, em geral, não comutam entre si, o que faz com que a ordem em que esses operadores são aplicados seja importante. O superpotencial, no caso geral, é dependente da variável espacial e de um ou mais parâmetros $\{a\}$ presentes no potencial.

Os operadores bosônicos, equações $(2)$ e $(3)$, são utilizados para fatorizar o Hamiltoniano $H$, dado pela equação (1), da seguinte forma:

$$
H=A^{+} A^{-}+E_{0},
$$

onde $E_{0}$ é o autovalor do nível mais baixo de energia do Hamiltoniano $H$.

Usando os operadores bosônicos na ordem estipulada para fatorizar $H$, reescrevemos a equação (4) como:

$$
H=-\frac{d^{2}}{d x^{2}}+W^{2}(x ; a)-\frac{d}{d x} W(x ; a)+E_{0} .
$$

Observa-se que no superpotencial $W(x ; a)$, é indicado de forma explícita a dependência na variável $x$ e de um conjunto de parâmetros $\{a\}$. Não há restrição ao número de parâmetros a serem introduzidos. Entretanto, para melhor compreensão do processo vamos restringir o formalismo ao uso de apenas um parâmetro. Além disso, um parâmetro é suficiente para trabalhar o problema sob análise. Usando a equação (5) e a definição de $H$, equação (1), obtemos a seguinte equação diferencial:

$$
V(x)-E_{0}=W^{2}(x ; a)-\frac{d}{d x} W(x ; a),
$$

que é um caso particular da equação de Ricatti [29]. A resolução da equação (6) permite identificar o superpotencial $W(x ; a)$ e o autovalor $E_{0}$ do menor nível de energia do Hamiltoniano $H$, o estado fundamental.

Uma vez obtido o superpotencial, podemos definir dois operadores Hamiltonianos, $H^{+}$e $H^{-}$, que são chamados de Hamiltonianos companheiros supersimétricos:

$$
\begin{aligned}
H^{+}=A^{+} A^{-} & =-\frac{d^{2}}{d x^{2}}+W^{2}(x ; a)-\frac{d}{d x} W(x ; a) \\
& =-\frac{d^{2}}{d x^{2}}+\mathrm{V}^{+}(x ; a), \\
H^{-}=A^{-} A^{+} & =-\frac{d^{2}}{d x^{2}}+W^{2}(x ; a)+\frac{d}{d x} W(x ; a) \\
& =-\frac{d^{2}}{d x^{2}}+\mathrm{V}^{-}(x ; a) .
\end{aligned}
$$

A construção indicada para o dois Hamiltonianos acima pode ser estendida para encontrar toda uma família de Hamiltonianos (hierarquia), relacionados entre si pela supersimetria $[4,5,30]$. Para a construção dos operadores escada, é necessário identificar a condição de shape invariance [4-6] do potencial estudado usando a definição dos Hamiltonianos companheiros supersimétricos, equações (7) e (8). O sistema é dito shape invariant se a diferença entre Hamiltonianos $H^{+}$e $H^{-}$for independente da coordenada espacial e obedecer a seguinte relação:

$$
R\left(a_{1}\right)=H^{-}-H^{+}=\mathrm{V}^{-}\left(x ; a_{0}\right)-\mathrm{V}^{+}\left(x ; a_{1}\right) .
$$


Os principais potenciais de interesse físico e que são shape invariant possuem parâmetros relacionados entre si por uma translação [31,32]. Assim, os parâmetros a podem ser relacionados pela adição de uma constante de translação $\eta$, ou seja, $a_{1}=a_{0}+\eta$. Por construção, a álgebra utilizada permite estender a relação entre os parâmetros para outros membros da hierarquia de Hamiltoniamos, i.e., $a_{2}=a_{1}+\eta, a_{3}=a_{2}+\eta$ etc. Dessa construção, podemos obter a relação entre um dado parâmetro $a_{k}$ e o parâmetro inicial observando todas as $k$ vezes em que o parâmetro foi transladado. Seguindo esse raciocínio, obtemos de forma direta a relação entre os parâmetros:

$$
a_{k}=a_{0}+k \eta
$$

Além disso, para potenciais shape invariant, cujos parâmetros são relacionados através de uma translação, podemos definir operadores-escada generalizados através de uma translação nos parâmetros e do uso dos operadores bosônicos, dados pelas equações (2) e (3), da seguinte forma:

$$
\begin{gathered}
B^{+}\left(a_{0}\right)=A^{+}\left(a_{0}\right) T^{+}\left(a_{0}\right)=A^{+}\left(a_{0}\right) e^{\eta \frac{\partial}{\partial a_{0}}}, \\
B^{-}\left(a_{0}\right)=T^{-}\left(a_{0}\right) A^{-}\left(a_{0}\right)=e^{-\eta \frac{\partial}{\partial a_{0}}} A^{-}\left(a_{0}\right)
\end{gathered}
$$

Esses operadores, equações 11) e 12, obedecem a estrutura algébrica necessária para identificá-los como operadores-escada [31-33], podendo ser associados com uma generalização dos operadores de criação e destruição utilizados em soluções algébricas do oscilador harmônico [2]. A título de recordação é oportuno lembrar que os operadores escadas são bastante estudados para o oscilador harmônico. A álgebra associada a esse caso pode ser expressa por $\left[a^{-}, a^{+}\right]=1 ;\left[\mathbf{N}, a^{+}\right]=a^{+} ;\left[\mathbf{N}, a^{-}\right]=-a^{-}$; sendo que $\mathbf{N},=a^{+} a^{-}$, com $a^{+}$e $a^{-}$representando os operadores de criação e destruição, respectivamente $[3,34]$.

Seguindo o tratamento usual, o operador de destruição aplicado ao estado fundamental permite a obtenção da função de onda deste estado fundamental $\psi_{0}$. Nesse caso, temos:

$$
B^{-}\left(a_{0}\right) \psi_{0}\left(x ; a_{0}\right)=T^{-}\left(a_{0}\right) A^{-}\left(a_{0}\right) \psi_{0}\left(x ; a_{0}\right)=0 .
$$

Uma vez que o operador de translação atua apenas no espaço de parâmetros da função de onda, para a igualdade da equação (13) ser verdadeira é suficiente que $A^{-}\left(a_{0}\right) \psi_{0}\left(x ; a_{0}\right)=0$. Assim, somos conduzidos ao resultado $[4,32]$ :

$$
\psi_{0}\left(x ; a_{0}\right)=N \exp \left[-\int_{x} W\left(\bar{x} ; a_{0}\right) d \bar{x}\right]
$$

sendo que $N$ é a constante de normalização.

O uso dos operadores-escada generalizados, equações (11) e (12), permite obter as autofunções e os autovalores de energia para os Hamiltonianos que possuem a propriedade de shape invariance. Isso é feito, em linhas gerais, usando as relações [4-6,28]:

$$
\begin{aligned}
\psi_{n}\left(x ; a_{0}\right) & =\left[\mathrm{B}^{+}\left(a_{0}\right)\right]^{n} \psi_{0}\left(x ; a_{0}\right), \\
E_{n} & =E_{0}+\sum_{i=1}^{n} R\left(a_{i}\right),
\end{aligned}
$$

onde $E_{0}$ é a constante subtraída do Hamiltoniano original ao fatorizarmos em termos dos operadores bosônicos, equação (4), e corresponde ao autovalor de energia do estado fundamental do sistema quântico estudado. O termo $R\left(a_{i}\right)$ é o resíduo que surge devido à propriedade de shape invariance, equação (9), com a translação no parâmetro dada pela equação (10). A autofunção do estado fundamental é determinada pela equação (14). Assim, identificando o superpotencial $W\left(x ; a_{0}\right)$ e o resíduo $R\left(a_{i}\right)$ é possível resolver o problema quântico sob análise.

\section{Separação de variáveis}

A equação de Schrödinger independente do tempo é uma equação diferencial de segunda ordem que pode ser escrita como [1-3]:

$$
-\nabla^{2} \psi(x, y, z)+V(x, y, z) \psi(x, y, z)=E \psi(x, y, z) .
$$

Lembrando que adotamos $\hbar=2 \mu=1$. $E$ é o autovalor da equação e corresponde a energia do sistema no estado descrito pela autofunção $\psi$

Para sistemas que possuem simetria esférica em que o potencial é função apenas da distância da origem do sistema de coordenadas até um certo ponto em $r$ (potenciais de força central), como é o caso do átomo de hidrogênio, convém transformar as coordenas espaciais do sistema cartesiano para o sistema esférico. Com essa mudança, o laplaciano $\nabla^{2}$ toma a forma [35]:

$$
\begin{aligned}
\nabla^{2} & =\frac{1}{r^{2}} \frac{\partial}{\partial r}\left(r^{2} \frac{\partial}{\partial r}\right)+\frac{1}{r^{2} \operatorname{sen} \theta} \frac{\partial}{\partial \theta}\left(\operatorname{sen} \theta \frac{\partial}{\partial \theta}\right) \\
& +\frac{1}{r^{2} \operatorname{sen}^{2} \theta}\left(\frac{\partial^{2}}{\partial \varphi^{2}}\right) .
\end{aligned}
$$

Substituindo a equação (18) na equação 17 , obtemos a equação de Schrödinger independente do tempo em coordenadas esféricas:

$$
\begin{aligned}
& -\left[\frac{1}{r^{2}} \frac{\partial}{\partial r}\left(r^{2} \frac{\partial}{\partial r}\right)+\frac{1}{r^{2} \operatorname{sen} \theta} \frac{\partial}{\partial \theta}\left(\operatorname{sen} \theta \frac{\partial}{\partial \theta}\right)\right. \\
& \left.+\frac{1}{r^{2} \operatorname{sen}^{2} \theta}\left(\frac{\partial^{2}}{\partial \varphi^{2}}\right)\right] \psi(r, \theta, \varphi)+V(r) \psi(r, \theta, \varphi) \\
& =E \psi(r, \theta, \varphi) .
\end{aligned}
$$

O potencial usado para descrever o átomo de hidrogênio é o potencial Coulombiano que vamos adotar aqui como sendo $V(r)=-1 / r$. Novamente, as constantes são adotadas como sendo iguais a 1, para não carregar a notação, embora a forma completa possa ser usada diretamente $V(r)=-\frac{e^{2}}{4 \pi \epsilon_{0} r}$ 
A equação (19) permite separação de variáveis, como é encontrado em diversos livros introdutórios de Mecânica Quântica (vide, por exemplo, [1] e [2]). O processo de separação de variáveis se inicia com a definição da função $\psi(r, \theta, \varphi)$ como sendo o produto de funções em cada uma das variáveis, $R(r), \Theta(\theta)$ e $\Phi(\varphi)$, ou seja,

$$
\psi(r, \theta, \varphi)=N R(r) \Theta(\theta) \Phi(\varphi) .
$$

sendo que $N$ é a constante de normalização. O resultado da separação de variáveis leva a três equações diferencias:

$$
\begin{gathered}
\frac{1}{r^{2}} \frac{d}{d r}\left(r^{2} \frac{d}{d r} R(r)\right)+\left\{[E-V(r)]-\frac{l(l+1)}{r^{2}}\right\} R(r)=0, \\
\frac{1}{\operatorname{sen} \theta} \frac{d}{d \theta}\left(\operatorname{sen} \theta \frac{d}{d \theta} \Theta(\theta)\right)+\left(l(l+1)-\frac{m^{2}}{\operatorname{sen}^{2} \theta}\right) \Theta(\theta)=0, \\
\frac{1}{\Phi(\varphi)} \frac{d^{2}}{d \varphi^{2}} \Phi(\varphi)=-m^{2}
\end{gathered}
$$

Como usual, três números quânticos são introduzidos, $m, l$ e $n$, relacionados com as constantes de separação das equações. Em particular, o número quântico $n$ está relacionado com a energia do sistema $E_{n}$ como indicado mais à frente.

As equações 21), 22 e 23 podem ser resolvidas de maneira tradicional, como encontrado nos livros [1-3]. Entretanto, exploramos uma abordagem alternativa, seguindo a metodologia mostrada na seção anterior.

\section{A equação na coordenada angular $\varphi$}

A equação em $\varphi$, equação 23 , pode ser reescrita como:

$$
-\frac{d^{2}}{d \varphi^{2}} \Phi(\varphi)=m^{2} \Phi(\varphi)
$$

Apesar de se tratar de uma equação diferencial simples, resolvê-la pelo formalismo supersimétrico requer cuidado para não cometer erros na abordagem matemática e/ou na interpretação física. De início, é bom frisar que, nesse caso, a invariância na forma funcional está escondida ( $h i d$ den shape invariance) [18], sendo necessário uma análise mais profunda da fatorização da equação diferencial.

A equação (24), constituída pela derivada de segunda ordem em $\varphi$, é formalmente semelhante ao Hamiltoniano de uma partícula confinada em um poço de potencial infinito ou finito unidimensional. Esse problema unidimensional tem sido extensamente analisado na literatura [1-3], inclusive via formalismo da Mecânica Quântica Supersimétrica $[4,36]$. Entretanto, o significa físico do problema de uma partícula em uma caixa unidimensional é distinto do caso estudado aqui que corresponde ao problema angular de sistema tridimensional. A diferença mais imediata diz respeito à condição de contorno adotada. Enquanto a partícula na caixa deve satisfazer a condição $\Phi(0)=\Phi(2 \pi)=0$, o problema angular impõe que $\Phi(\varphi)=\Phi(\varphi+2 \pi)$ Essa nova condição muda a caracterização do problema e, por conseguinte, a sua solução matemática.

Seguindo o método proposto, a fatorização da equação (24) leva a uma expressão similar à equação (5):

$$
H=-\frac{d^{2}}{d \varphi^{2}}+W^{2}(\varphi ; a)-\frac{d}{d \varphi} W(\varphi ; a)+m_{0}^{2} .
$$

Neste ponto, é oportuno destacar que $m^{2}$ representa os autovalores da equação 24, mas não corresponde à energia, uma vez que os níveis de energia para o átomo de hidrogênio são obtidos dos autovalores da equação diferencial na coordenada radial [1-3].

Observa-se que um superpotencial constante satisfaz a equação de Ricatti, equação (6), porém essa escolha leva a problemas na construção da shape invariance dificultando a construção dos operadores escada. Outra forma para o superpotencial que satisfaz a equação de Ricatti para esse problema é dada por:

$$
W\left(\varphi ; a_{0}\right)=a_{0} \tan ^{2}(\varphi) .
$$

Com essa escolha, a equação 25 e seu companheiro supersimétrico, equação (8), tornam-se:

$$
\begin{aligned}
& H^{+}=-\frac{d^{2}}{d \varphi^{2}}+a_{0}\left(a_{0}-1\right) \sec ^{2}(\varphi)-a_{0}^{2}, \\
& H^{-}=-\frac{d^{2}}{d \varphi^{2}}+a_{0}\left(a_{0}+1\right) \sec ^{2}(\varphi)-a_{0}^{2} .
\end{aligned}
$$

Nota-se que se tomarmos $a_{0}=1$ ou $a_{0}=0$ ao usarmos o Hamiltoniano descrito pela equação (27) na fatorização do potencial original obtemos que $m_{0}^{2}$ é igual a um ou zero e identificamos a invariância na forma funcional do potencial com o respectivo resíduo, equação (9):

$$
R\left(a_{1}\right)=\left[a_{0}\left(a_{0}+1\right)-a_{1}\left(a_{1}-1\right)\right] \sec ^{2}(\varphi)+a_{1}^{2}-a_{0}^{2} .
$$

É necessário cuidado ao tratar com esse problema, pois usar $a_{0}=1$ no início do processo, na definição do superpotencial, equação (26), pode levar a pressupor que o sistema não é shape invariance. Esse assunto já foi discutido na literatura [18] tendo sido identificada uma shape invariance escondida neste tipo de problema. Essa shape invariance fica evidente ao se deixar indicada a dependência do superpotencial com o parâmetro $a_{0}$.

Para esse caso, a exigência de que a equação (29) seja independente da variável espacial devido a condição de shape invariance, equação (9), impõe que:

$$
\left[a_{0}\left(a_{0}+1\right)-a_{1}\left(a_{1}-1\right)\right]=0 .
$$

Esta condição é satisfeita se $a_{1}=a_{0}+1$ ou $a_{1}=-a_{0}$. Desta forma, se $a_{0}$ for igual a zero, então $a_{1}=0$ também. Caso $a_{0}=1$ então $a_{1}$ vale 2 ou -1 . Desta forma, a equação 29 fornece:

$$
R\left(a_{1}\right)=\left\{\begin{array}{l}
2 a_{0}+1, a_{0}=1 \\
0, a_{0}=0
\end{array} .\right.
$$


Generalizando os resíduos usando a equação 10 e usando o parâmetro de translação $\eta=1$ se $a_{0}=1$, e $\eta=0$ para o caso em que $a_{0}=0$, obtemos:

$$
R\left(a_{k}\right)=\left\{\begin{array}{l}
2 \mathrm{k}+1, a_{0}=1 \\
0, a_{0}=0
\end{array} .\right.
$$

É possível identificar $m_{0}$ na equação 27 como sendo igual a $a_{0}$. No caso em que $a_{0}=1$, a equação para os autovalores, dada pela substituição da equação 32 e do autovalor $m_{0}^{2}$ na equação 16 , nos fornece:

$$
m_{n}^{2}=\mathrm{m}_{0}^{2}+\sum_{k=1}^{n}(2 k+1)=(n+1)^{2} .
$$

Já quando $a_{0}=0$ caímos no caso trivial em que $m^{2}$ é igual a zero.

Como consequência do formalismo utilizado, observamos que mé um número inteiro, não sendo mais necessário o uso do subíndice $n$, ou seja, $m=0, \pm 1, \pm 2, \pm 3 \ldots$, sendo que o sinal \pm aparece naturalmente quando se aplica a raiz quadrada aos dois membros da equação (33) $\left(m^{2}=(n+1)^{2}\right)$. Como esperado, esse resultado é o mesmo que é indicado pelo uso de outros métodos [1-2].

Com relação à autofunção, podemos obtê-la através da aplicação do superpotencial na equação (14):

$$
\Phi_{0}\left(\varphi ; a_{0}\right) \propto \cos ^{a_{0}}(\varphi) .
$$

Uma vez que conhecemos o superpotencial, equação (26), o parâmetro da shape invariance, $\eta$ e a autofunção para o mais baixo estado, equação (34), podemos construir os operadores-escada generalizados e determinar os outros estados a partir do primeiro. Neste caso, os operadores de criação e destruição são dados por:

$$
\begin{aligned}
& B^{+}\left(a_{0}\right)=\left[-\frac{d}{d \varphi}+a_{0} \tan (\varphi)\right] \exp \left[\eta \frac{\partial}{\partial a_{0}}\right], \\
& B^{-}\left(a_{0}\right)=\exp \left[-\eta \frac{\partial}{\partial a_{0}}\right]\left[\frac{d}{d \varphi}+a_{0} \tan (\varphi)\right] .
\end{aligned}
$$

Aplicando o operador de criação, equação (35), na autofunção dada pela equação (34), obtemos a função, a menos da constante de normalização:

$$
\Phi_{1}\left(\varphi ; a_{0}\right) \propto \cos ^{a_{0}}(2 \varphi) .
$$

Aplicando $|m|$ vezes o operador à autofunção (34 obtemos a autofunção para o m-ésimo termo, ou seja:

$$
\Phi_{n}\left(\varphi ; a_{0}\right) \propto \cos ^{a_{0}}(m \varphi) \operatorname{com} m=1,2,3 \ldots \text { e } a_{0}=1 .
$$

Devemos lembrar também de incluir o caso em que $a_{0}=m=0$ como uma possível solução. Nesse caso, a autofunção é constante e o autovalor é igual a zero.

Apesar do procedimento adotado estar correto, as autofunções indicadas na equação (38) ainda não representam a solução completa do problema de resolver a equação 24. Isto porque a equação de Ricatti associada ao Hamiltoniano 25 possui ainda uma outra solução independente. Essa solução é:

$$
W\left(\varphi ; a_{0}\right)=-a_{0} \cot (\varphi) .
$$

Usando esse superpotencial os Hamiltonianos companheiros supersimétricos passam a ser:

$$
\begin{aligned}
& H^{+}=-\frac{d^{2}}{d \varphi^{2}}+a_{0}\left(a_{0}-1\right) \csc ^{2}(\varphi)-a_{0}^{2}, \\
& H^{-}=-\frac{d^{2}}{d \varphi^{2}}+a_{0}\left(a_{0}+1\right) \csc ^{2}(\varphi)-a_{0}^{2} .
\end{aligned}
$$

A condição de shape invariance é muito similar àquela obtida para o superpotencial usado anteriormente:

$$
R\left(a_{1}\right)=\left[a_{0}\left(a_{0}+1\right)-a_{1}\left(a_{1}-1\right)\right] \csc ^{2}(\varphi)+a_{1}^{2}-a_{0}^{2} .
$$

O método de solução exige que a equação (42) seja independente da variável espacial, ou seja:

$$
\left[a_{0}\left(a_{0}+1\right)-a_{1}\left(a_{1}-1\right)\right]=0 .
$$

As mesmas condições anteriores devem ser satisfeitas, ou seja, $a_{1}=a_{0}+1$ ou $a_{1}=-a_{0}$. Essas condições levam ao mesmo valor para o parâmetro de translação, $\eta=1$ ou $\eta=0$, e o resíduo é o mesmo que o dado pela equação 31 . O raciocínio a ser empregado aqui é idêntico ao realizado anteriormente e, consequentemente, os autovalores são os mesmos, equação (33).

Com relação aos operadores-escada, o novo superpotencial nos traz:

$$
\begin{aligned}
& B^{+}\left(a_{0}\right)=\left[-\frac{d}{d \varphi}-a_{0} \cot (\varphi)\right] \exp \left[\eta \frac{\partial}{\partial a_{0}}\right], \\
& B^{-}\left(a_{0}\right)=\exp \left[-\eta \frac{\partial}{\partial a_{0}}\right]\left[\frac{d}{d \varphi}-a_{0} \cot (\varphi)\right] .
\end{aligned}
$$

A primeira autofunção é obtida pela aplicação do operador destruição a autofunção e igualado o resultado a zero. O resultado, em termos do superpotencial é expresso na equação (14). No presente caso, obtemos (a menos da normalização):

$$
\Phi_{0}\left(\varphi ; a_{0}\right) \propto \operatorname{sen}^{a_{0}}(\varphi) .
$$

A aplicação do operador de criação, equação 44, nessa autofunção $|m|$ vezes, fornece:

$$
\Phi_{n}\left(\varphi ; a_{0}\right) \propto \operatorname{sen}^{a_{0}}(m \varphi) \operatorname{com} m=1,2,3 \ldots e a_{0}=1 .
$$

Seguindo as noções básicas de resolução de equações diferenciais lineares [37], a solução geral para o problema é formada pela soma das soluções particulares. Assim, combinando as autofunções (38) e 47) obtemos uma autofunção que é a soma de cossenos e senos. Essa expressão pode ser reescrita como uma exponencial imaginária pela fórmula de Euler [38], ou seja:

$$
\Phi_{n}\left(\varphi ; a_{0}\right)=A_{1} \cos ^{a_{0}}(m \varphi)+A_{2} \operatorname{sen}^{a_{0}}(m \varphi) \propto e^{i m \varphi},
$$


com $a_{0}=1$ e $m=0, \pm 1, \pm 2, \pm 3 \ldots, A_{1}$ e $A_{2}$ são constantes. A forma exponencial da solução, expressa pela equação (48), é geralmente apresentada nos livros de Mecânica Quântica como solução da equação de Schrödinger na variável $\varphi$, sendo uma componente dos Harmônicos Esféricos $[1,2]$.

Como indicado anteriormente, o problema tratado nessa seção é similar àquele de uma partícula em uma caixa unidimensional. Entretanto, uma diferença importante está relacionada às condições de contorno que deve ser imposta à solução encontrada, como usual.

O problema da caixa unidimensional exige que as autofunções sejam nulas nos extremos da caixa o que, neste caso, equivale a impor que $\Phi(0)=\Phi(2 \pi)=[2]$. Por outro lado, a solução angular apresentada aqui deve satisfazer as condições de contorno periódicas $\Phi(\varphi)=\Phi(\varphi+2 \pi)$. Caso a solução indicada não possa satisfazer as condições de contorno, ela deve ser descartada e outra deve ser procurada. Entretanto, notamos que a solução apresentada, equação (48), satisfaz a condição periódica exigida no problema.

\section{A equação na coordenada angular $\theta$}

A equação diferencial na variável angular $\theta$, equação 22 , pode ser reescrita, usando a regra do produto para $\mathrm{O}$ primeiro termo do lado esquerdo, como:

$$
\begin{aligned}
& \frac{d^{2}}{d \theta^{2}} \Theta(\theta)+\cot (\theta) \frac{d}{d \theta} \Theta(\theta) \\
& +\left[l(l+1)-\frac{m^{2}}{\operatorname{sen}^{2}(\theta)}\right] \Theta(\theta)=0 .
\end{aligned}
$$

A solução dessa equação através do formalismo supersimétrico é indicada na referência [14], da qual seguimos uma parte do formalismo. Inicialmente, observamos que é possível eliminar o termo de primeira derivada desta equação fazendo uma mudança para uma nova variável, z. Para fazer esta mudança vamos introduzir a função de mapeamento $\theta=f(z)$ [15]. Assim, obtemos:

$$
\begin{aligned}
& \frac{d^{2}}{d z^{2}} \Theta(z)+\left[-\frac{f^{\prime \prime}(z)}{f^{\prime}(z)}+f^{\prime}(z) \cot [f(z)]\right] \frac{d}{d z} \Theta(z) \\
& +f^{\prime 2}(z)\left[l(l+1)-\frac{m^{2}}{\operatorname{sen}^{2}[f(z)]}\right] \Theta(z)=0
\end{aligned}
$$

Para eliminarmos o termo com a primeira derivada fazemos:

$$
-\frac{f^{\prime \prime}(z)}{f^{\prime}(z)}+f^{\prime}(z) \cot [f(z)]=0 .
$$

Pode-se verificar que a igualdade acima é satisfeita quando $f(z)=2 \operatorname{arctg}\left(e^{z}\right)$ [14], de modo que a equação (50) pode ser reescrita como:

$$
\left[-\frac{d^{2}}{d z^{2}}-l(l+1) \operatorname{sech}^{2}(z)\right] \Theta(z)=-m^{2} \Theta(z) .
$$

A equação (52) possui um formato conveniente para a realização da fatorização e também é similar ao da equação de Schrödinger para um caso particular do potencial de Rosen-Morse unidimensional [39], que já foi estudado através do formalismo da MQS [28,40], possuindo solução exata. Observamos que nesta equação as autofunções são dadas por $\Theta(z)$ e o autovalor é $-m^{2}$. Assim, partiremos da equação (52), usando um potencial da forma $V(z)=-V_{0} \operatorname{sech}^{2}(z)$, onde $V_{0}=l(l+1)$ Para esse caso, a fatorização possui a forma:

$$
\begin{gathered}
-\frac{d^{2}}{d z^{2}}-V_{0} \operatorname{sech}^{2}(z)=-\frac{d^{2}}{d z^{2}}+W^{2}\left(z ; a_{0}\right) \\
-\frac{d}{d z} W\left(z ; a_{0}\right)-m_{0}^{2} .
\end{gathered}
$$

O superpotencial que satisfaz essa equação de Ricatti para esse problema é conhecido na literatura e é dado por [28]:

$$
W\left(z ; a_{0}\right)=a_{0} \tanh (z) .
$$

Esse superpotencial conduz a $W^{2}\left(z ; a_{0}\right)=a_{0}^{2} \tanh ^{2}(z)=$ $a_{0}^{2}\left[1-\operatorname{sech}^{2}(z)\right]$ e $\frac{d}{d z} W\left(z ; a_{0}\right)=a_{0} \operatorname{sech}^{2}(z)$. Desse modo, a fatorização se torna:

$$
\begin{array}{r}
-\frac{d^{2}}{d z^{2}}-V_{0} \operatorname{sech}^{2}(z)=-\frac{d^{2}}{d z^{2}} \\
+a_{0}^{2}\left[1-\operatorname{sech}^{2}(z)\right]-a_{0} \operatorname{sech}^{2}(z)-m_{0}^{2},
\end{array}
$$

ou seja, o potencial original é relacionado com o superpotencial da seguinte forma:

$$
-V_{0} \operatorname{sech}^{2}(z)=a_{0}^{2}-\left(a_{0}^{2}+a_{0}\right) \operatorname{sech}^{2}(z)-m_{0}^{2} .
$$

Para que a igualdade da equação $(56)$ seja válida, devemos ter $V_{0}=\left(a_{0}^{2}+a_{0}\right)$ o que, pela definição: $V_{0}=l(l+1)$ permite identificar que $a_{0}=l$ ou $a_{0}=-l-1$. Além disso, o autovalor deve ser dado por:

$$
-m_{0}^{2}=-a_{0}^{2}
$$

A inversão na ordem de aplicação dos operadores bosônicos permite identificar a condição de shape invariance, através da equação (9). O resíduo é dado, então, por:

$$
R\left(a_{1}\right)=a_{0}^{2}-a_{1}^{2}+\left(a_{1}^{2}-a_{0}^{2}+a_{0}+a_{1}\right) \operatorname{sech}^{2}(z)
$$

Uma vez que a condição de shape invariance impõe que a equação (58) não pode depender da variável espacial $z$, devemos admitir que $a_{1}=a_{0}-1$. Outra solução possível é $a_{1}=-a_{0}$, mas esse caso não permite a translação do parâmetro e leva a uma autofunção não normalizável. Dessa forma, essa segunda opção deve ser descartada. Com a condição obtida para $a_{1}$ e a relação $a_{1}=a_{0}+\eta$, notamos que $\eta=-1$ e a equação 58 torna-se:

$$
R\left(a_{1}\right)=2 a_{1}+1 \text {. }
$$

O próximo passo da solução por meio desta metodologia é generalizar o resíduo por meio da equação (10). Nesse sentido, usando essa igualdade e a relação 
$a_{k}=a_{0}+k \eta$, equação 107 , obtemos $a_{k}=a_{0}-k$, uma vez que $\eta=-1$, o que permite a generalização dos resíduos:

$$
R\left(a_{k}\right)=a_{k-1}^{2}-a_{k}^{2}=2\left(a_{0}-k\right)+1 .
$$

A substituição das equações (57) e 60 na equação 16 permite determinar os autovalores da equação em $\theta$, que são:

$$
-m_{j}^{2}=-m_{0}^{2}+\sum_{k=1}^{j}\left[2\left(a_{0}-k\right)+1\right] .
$$

Substituindo a equação (57) na equação (61) e realizando a somatória até o j-ésimo termo, obtemos:

$$
-m_{j}^{2}=-\left(a_{0}-j\right)^{2} .
$$

Deve ser observado que a somatória de constantes é igual a própria constante multiplicada pelo número de termos da soma e que a somatória de termos proporcionais ao índice da soma se constitui de uma progressão aritmética de razão 1 . Usando a igualdade $a_{0}=l$ na equação 62 obtemos os autovalores de energia:

$$
-m_{j}^{2}=-(l-j)^{2} .
$$

A outra possibilidade obtida anteriormente, $a_{0}=-l-$ 1, também poderia ser usada. Entretanto, esta opção conduz a uma solução não normalizável o que implica que essa solução deve ser excluída.

O autovalor encontrado, ao ser comparado com o que é fornecido pela equação 52 , nos dá uma relação entre os parâmetros $(j, l$ e $m$ ) como sendo $m=l-j$ ou ainda, como indicado na ref. [14]:

$$
j=l-m \text {. }
$$

Observa-se que o índice $j$ pode ser suprimido da solução através desta igualdade. Também é importante observar que todos as quantidades envolvidas $(j, l, m)$ são números inteiros sendo que $j$ e $l$ são números naturais (inteiros maiores ou iguais a zero). Os valores permitidos de $m$ foram estabelecidos na seção anterior, $j$ vem do somatório presente na equação 61, portanto tem que ser um número natural, e $l$ escrito como a diferença de dois números inteiros, também deve ser inteiro. Observa-se ainda, através da equação (64), que $m \leq l$, pois, caso contrário, obteríamos um valor de $j<0$, que não é permitido, pois está relacionado a um índice da somatória na equação 61).

A autofunção relacionada ao menor autovalor da equação 52 , i.e., $m^{2}=a_{0}^{2}$ é obtida diretamente pela aplicação da equação (14), a menos da constante de normalização:

$$
\Theta_{0}\left(\mathrm{z} ; \mathrm{a}_{0}\right) \propto \operatorname{sech}^{a_{0}}(z) .
$$

É possível retornar à variável original $(\theta)$ através da relação $\theta \equiv f(z)=2 \operatorname{arctg}\left(e^{z}\right)$, obtida anteriormente. Esse cálculo foi realizado com substituição direta desta relação na autofunção (64). Para essa dedução usamos os recursos de cálculo analítico do software Wolfram Mathematica 11 e a relação $a_{0}=l$, o resultado obtido é:

$$
\Theta_{0}(\theta ; l) \propto \operatorname{sen}^{l}(\theta) .
$$

De forma análoga ao que foi feito na seção anterior, o próximo passo para resolver o problema sob análise é a determinação dos operadores-escada generalizados. Isso é feito identificado o superpotencial, equação (54), e os parâmetros ligados à shape invariance, $\eta$ e $a_{0}$. Esses operadores são escritos através da equação 11) e (12), e possuem a forma:

$$
\begin{gathered}
B^{+}(l)=\left[-\frac{d}{d z}+\operatorname{ltgh}(z)\right] \exp \left[-\frac{\partial}{\partial l}\right], \\
B^{-}(l)=\exp \left[\frac{\partial}{\partial l}\right]\left[\frac{d}{d z}+\operatorname{ltgh}(z)\right] .
\end{gathered}
$$

Aplicando o operador criação, equação (67), na autofunção (65), obtemos (a menos da constante de normalização):

$$
\Theta_{1}(z ; l)=(2 l-1) \operatorname{sech}^{l-1}(z) \operatorname{sen}(z) .
$$

Retornando à variável $\theta$, temos:

$$
\Theta_{1}(\theta ; l)=(1-2 l) \operatorname{sen}^{l-1}(\theta) \cos (\theta) .
$$

Notamos que são necessários apenas dois números inteiros, $l$ e $m$, para caracterizar a autofunção, o número natural $j$ é fixado pelo vínculo expresso na equação (64). Uma vez respeitado esse vínculo, os resultados aqui obtidos são similares aos resultados obtidos por outros métodos [1-3], como observado ao comparar as autofunções para diferentes valores dos parâmetros $l, m$ e $j$, dadas pelas equações (66) e (70), com as funções associadas de Legendre.

Devido ao vínculo apresentado pela equação (64), as autofunções que possuem os menores autovalores são àquelas em que $m=l$, enquanto as outras autofunções são obtidas pela aplicação do operador de criação, equação 67, nessas funções. Podemos então relacionar, de maneira generalizada, as autofunções $\Theta_{j}(\theta ; l)$ com as funções associadas de Legendre $P_{l}^{l-j}(\cos \theta)$. O primeiro caso corresponde a $l=0$ e $m=0$, cuja autofunção é dada pela equação $(66)$ com $l=0$. Sempre que $l$ for igual a $m$ a autofunção é obtida usando a equação (66). Na Tabela 1 isso corresponde a $l=m=1, l=m=2$, $l=m=3$. Usando os números $l=1$ e $m=0$ obtemos a autofunção aplicando o operador de criação, equação (67), a autofunção correspondente a $l=1$ e $m=1$. Esse operador também é aplicado nas autofunções correspondentes a $l=2$ e $m=2 ; l=3$ e $m=3$ para obter as autofunções correspondentes à $l=2$ e $m=1 ; l=3 \mathrm{e}$ $m=2$, respectivamente.

A Tabela 1 apresenta um quadro comparativo entre alguns resultados obtidos pela metodologia aqui apresentada e aqueles indicados na literatura. Como esperado, observa-se que os resultados são os mesmos. 
Tabela 1: Algumas das Funções Associadas de Legendre, ref. [2], e os resultados obtidos pela abordagem desenvolvida aqui

\begin{tabular}{lllll}
\hline $\mathbf{j}$ & $\mathbf{l}$ & $\mathbf{m}$ & $\mathbf{P}_{\mathbf{1}}^{\mathbf{m}}(\mathbf{c o s} \theta)[\mathbf{2}]$ & $\boldsymbol{\Theta}_{\mathbf{j}}(\theta ; \mathbf{l})$ \\
\hline 0 & 0 & 0 & 1 & 1 \\
0 & 1 & 1 & $\operatorname{sen}(\theta)$ & $\operatorname{sen}(\theta)$ \\
0 & 2 & 2 & $3 \operatorname{sen}^{2}(\theta)$ & $\operatorname{sen}^{2}(\theta)$ \\
0 & 3 & 3 & $15 \operatorname{sen}(\theta)\left[1-\cos ^{2}(\theta)\right]$ & $\operatorname{sen}^{3}(\theta)=\operatorname{sen}(\theta)\left[1-\cos ^{2}(\theta)\right]$ \\
1 & 1 & 0 & $\cos (\theta)$ & $\cos (\theta)$ \\
1 & 2 & 1 & $3 \operatorname{sen}(\theta) \cos (\theta)$ & $\operatorname{sen}(\theta) \cos (\theta)$ \\
1 & 3 & 2 & $15 \operatorname{sen}^{2}(\theta) \cos (\theta)$ & $\operatorname{sen}^{2}(\theta) \cos (\theta)$
\end{tabular}

É oportuno destacar a ausência das constantes multiplicando as funções trigonométricas exibidas na coluna $\Theta_{j}(\theta ; l)$ da Tabela 1 devido ao fato de que, na abordagem adotada, a normalização é realizada em separado, ao final do processo, como argumentado anteriormente.

\section{A equação na coordenada radial}

A equação diferencial radial para o átomo de hidrogênio é dada pela equação (21). Lembrando que a letra $l$ corresponde ao número quântico do momento angular, já discutido na seção anterior. Introduzindo uma nova função $f(r)$, tal que $R(r)=f(r) / r$, a derivada primeira pode ser eliminada da equação (21). Esta equação pode ser reescrita como:

$$
\frac{d^{2}}{d r^{2}} f+V_{e f}(r) f=E f .
$$

onde o potencial efetivo, $V_{e f}(r)$, inclui o termo coulombiano e o termo de barreira de potencial:

$$
V_{e f}(r)=-\frac{1}{r}+\frac{l(l+1)}{r^{2}} .
$$

Assim, o Hamiltoniano original relacionado com a coordenada radial é escrito como:

$$
H=-\frac{d^{2}}{d r^{2}}-\frac{1}{r}+\frac{l(l+1)}{r^{2}} .
$$

O superpotencial, neste caso, é obtido através da fatorização do Hamiltoniano original o que leva a necessidade de resolver a equação de Ricatti, equação (6). Para esse problema específico, o superpotencial é conhecido [4] e dado por:

$$
W(r ; l)=\frac{1}{2(l+1)}-\frac{l+1}{r}
$$

Com este superpotencial o Hamiltoniano é fatorizando, equação (7), fornecendo:

$$
\begin{aligned}
& H^{+}=A^{+} A^{-}= \\
& -\frac{d^{2}}{d r^{2}}-\frac{1}{r}+\frac{l(l+1)}{r^{2}}+\frac{1}{4(l+1)^{2}}=H-E_{0}^{(l)} .
\end{aligned}
$$

A fim de verificar se esse potencial é shape invariant obtemos o companheiro supersimétrico do Hamiltoniano
$H^{+}$, equação 75$)$, usando a definição de $H^{-}$indicada na equação 8. Desse modo, temos:

$$
\begin{gathered}
H^{-}=-\frac{d^{2}}{d r^{2}}+W^{2}(r ; l)+\frac{d}{d r} W(r ; l)= \\
-\frac{d^{2}}{d r^{2}}-\frac{1}{r}+\frac{(l+1)(l+2)}{r^{2}}+\frac{1}{4(l+1)^{2}} .
\end{gathered}
$$

Portanto, obtemos:

$$
V^{+}(r)=-\frac{1}{r}+\frac{l(l+1)}{r^{2}}+\frac{1}{4(l+1)^{2}}
$$

$$
V^{-}(r)=-\frac{1}{r}+\frac{(l+1)(l+2)}{r^{2}}+\frac{1}{4(l+1)^{2}} .
$$

Comparando a estrutura dos potenciais dos companheiros supersimétricos, equações (77) e (78), podemos identificar o número quântico $l$ como sendo o parâmetro da shape invariance. Seguindo essa linha, vamos introduzir a notação $a_{0}=l_{0}$.

Assim, a condição de invariância da forma, equação (9), para a equação radial usando o potencial coulombiano fornece:

$$
\begin{aligned}
R\left(l_{1}\right)= & V^{-}\left(r ; l_{0}\right)-V^{+}\left(r ; l_{1}\right)=\frac{\left(l_{0}+1\right)\left(l_{0}+2\right)}{r^{2}} \\
& +\frac{1}{4\left(l_{0}+1\right)^{2}}-\frac{l_{1}\left(l_{1}+1\right)}{r^{2}}-\frac{1}{4\left(l_{1}+1\right)^{2}} .
\end{aligned}
$$

Para que $R\left(l_{1}\right)$ seja independente da coordenada $r$, a equação 79 fornece $l_{1}=l_{0}+1$ e o resíduo $R\left(l_{1}\right)$ acima é reescrito como:

$$
R\left(l_{1}\right)=\frac{1}{4\left(l_{0}+1\right)^{2}}-\frac{1}{4\left(l_{1}+1\right)^{2}} .
$$

Outra possibilidade seria fazer $l_{1}=-l_{0}-2$, mas essa condição leva a uma autofunção não normalizável e deve ser descartada. Desta forma, usando a relação $l_{1}=l_{0}+1$ e equação (10), com $k=1$, obtemos o valor do parâmetro de translação $\eta=1$.

A expressão do resíduo, equação 80 , pode ser generalizada para $k$ translações. Usando a equação 10 com $\eta=1$ obtemos $l_{k}=l_{0}+k$. O resíduo, neste caso, é escrito:

$$
\begin{aligned}
R\left(l_{k}\right) & =\frac{1}{4}\left[\frac{1}{\left(l_{k-1}+1\right)^{2}}-\frac{1}{\left(l_{k}+1\right)^{2}}\right] \\
& =\frac{1}{4}\left[\frac{1}{\left(l_{0}+k\right)^{2}}-\frac{1}{\left(l_{0}+k+1\right)^{2}}\right] .
\end{aligned}
$$


Os autovalores de energia são encontrados usando a equação 16. Lembrando que a energia do estado fundamental $E_{0}^{(l)}$ é dado pelo termo constante na definição do primeiro Hamiltoniano companheiro supersimétrico, equação 75 , o que conduz a:

$$
E_{0}^{(l)}=-\frac{1}{4(l+1)^{2}} .
$$

Na sequência, podemos encontrar os níveis de energia substituindo na equação 16 as equações (81) e 82 :

$$
E_{n}^{(l)}=E_{0}^{(l)}+\sum_{k=1}^{n} R\left(l_{k}\right)=-\frac{1}{4(l+n+1)^{2}} .
$$

com $n=1,2,3 \ldots$ Para chegar a esse resultado, basta abrir a somatória e observar que todos os termos se cancelam, exceto o primeiro e o último, o que conduz ao resultado mostrado na equação 83 .

Em seguida obtemos os operadores-escada generalizados de acordo com as equações (11) e (12). Para o problema radial do potencial de Coulomb, esses operadores são:

$$
B^{+}(l)=\left[-\frac{d}{d r}+\frac{1}{2(l+1)}-\frac{(l+1)}{r}\right] \exp \left(\frac{\partial}{\partial l}\right)
$$

e

$$
B^{-}(l)=\exp \left(-\frac{\partial}{\partial l}\right)\left[\frac{d}{d r}+\frac{1}{2(l+1)}-\frac{(l+1)}{r}\right] .
$$

As autofunções dos níveis de mais baixa energia para cada valor de $l$ são obtidas aplicando-se o operador destruição 85 como indicado na relação 13 ou, da mesma forma substituindo-se o superpotencial (74) na relação (14), o que fornece:

$$
\psi_{0}^{(l)}(r) \propto r^{l+1} e^{-\frac{r}{2(l+1)}}
$$

As outras autofunções, para $n \geq 1$, são calculadas pela ação do operador de criação 84 aplicado na autofunção dada pela equação 86 . Por exemplo, para $n=1$ obtemos as seguintes autofunções:

$$
\psi_{1}^{(l)}(r) \propto\left\{\left[\frac{1}{l+2}+\frac{1}{l+1}\right] r-2 l-3\right\} r^{l+1} e^{-\frac{r}{2(l+2)}} .
$$

A expressão exibida na equação 87) é a mesma encontrada usando o método de Frobenius [1-3] para $n=1$ e $l$ podendo variar para qualquer número inteiro maior ou igual a 1.

De forma geral, os resultados obtidos aqui, autovalores e autofunções para a parte radial da equação de Schrödinger, são os mesmos obtidos por outros métodos [1-3]. Vale salientar que no problema coulombiano, o número quântico principal normalmente utilizado para descrever um estado quântico é $N=n+l+1$

\section{Conclusão}

Apresentamos um método alternativo para obter as autofunções e autovalores da equação de Schrödinger para o átomo de hidrogênio em coordenadas esféricas. A abordagem adotada envolve a identificação de operadores de criação e destruição usando o formalismo da supersimetria em mecânica quântica e a propriedade de shape invariance.

A solução geral das autofunções é obtida pela equação 20 com as autofunções obtidas para cada coordenada individual. Os autovalores de energia são dados pela equação (83), obedecendo-se os vínculos estabelecidos entre os números quânticos que surgem do formalismo.

No caso da equação diferencial na coordenada $\varphi$, a condição de shape invariance não é obtida diretamente e é caracterizada como uma shape invariance escondida [18]. A solução geral para essa equação, escrita como uma função exponencial, coincide com as soluções apresentadas em outras abordagens $[1,2]$. As equações nas outras coordenadas seguem o mesmo caminho obtendo as soluções iguais àquelas encontradas por outros métodos [1-3].

Uma vez discutido o átomo de hidrogênio, abre-se a possibilidade de usar o formalismo para estudar outros problemas quânticos, o que indica que tratamentos alternativos podem ser aplicados neste contexto.

\section{Agradecimentos}

Os autores agradecem o apoio parcial recebido do Conselho Nacional de Desenvolvimento Científico e Tecnológico $(\mathrm{CNPq})$. JMCM agradece à Coordenação de Aperfeiçoamento de Pessoal de Nível Superior (CAPES) pelo apoio, e AAJ agradece o apoio de um ano da CAPES

\section{Referências}

[1] L.I. Schiff, Quantum Mechanics (McGraw-Hill Book Company, New York, 1949), $3^{\text {a }}$ ed.

[2] D.J. Griffiths, Introduction to Quantum Mechanics (Pearson Prentice Hall, New Jersey, 2004).

[3] A.S. Davydov, Quantum Mechanics: International Series in Natural Philosophy, (Pergamon, Massachusetts, 1968), $2^{\mathrm{a}}$ ed.

[4] E. Drigo Filho, Supersimetria Aplicada à Mecânica Quântica: Estudo da Equação de Schrödinger (Editora UNESP, São Paulo, 2009).

[5] F. Cooper, A. Khare e U. Sukhatme, Phys. Rep. 251, 267 (1995).

[6] G. Junker, Supersymmetric Methods in Quantum and Statistical Physics (Springer-Verlag Berlin Heidelberg, Heidelberg, 1996).

[7] D. DeLaney e M.M. Nieto, Phys. Lett. B 247, 301 (1990).

[8] J. Bougie, A. Gangopadhyaya e C. Rasinariu, J. Phys. A: Math. Theor. 51, 375202 (2018).

[9] J.C.B. Araujo, G.R. Borges e E. Drigo Filho, Rev. Bras. Ens. Fís. 28, 41 (2006). 
[10] E. Drigo Filho e R.M. Ricotta, Tren. App. Comp. Math. 6, 73 (2005)

[11] E. Drigo Filho e R.M. Ricotta, Mod. Phys. Lett. A 10, 1613 (1995).

[12] A. Vega e J. Flores, Pramana 87, 73 (2016).

[13] J.O. Rosas-Ortiz, J. Phys. A: Math. Gen. 31, 10163 (1998).

[14] R. Dutt, A. Gangopadhyaya e U.P. Sukhatme, Am. J. Phys. 65, 400 (1997).

[15] D. Bazeia e A. Das, Phys. Lett. B 715, 256 (2012).

[16] F. Cooper, A. Khare e U. Sukhatme, Phys. Rep. 251, 267 (1995).

[17] N.A. Alves e E. Drigo Filho, J. Phys. A: Math. Gen. 21, 3215 (1988)

[18] E. Drigo Filho e M.R. Ricotta, J. Phys. A: Math. Gen. 37, 10057 (2004).

[19] G. Levai, J. Phys. A: Math. Gen. 22, 689 (1989).

[20] C.S. Jia, J.Y. Wang, S. He e L.T. Sun, J. Phys. A: Math. Gen. 33, 6993 (2000).

[21] S.W. Qian, B.W. Huang e Z.Y. Gu, New J. Phys. 4, 13 (2002).

[22] B. Bagchi, A. Banerjee, C. Quesne e V.M. Tkachuk, J. Phys. A: Math. Gen. 38, 2929 (2005)

[23] A. Ganguly e L.M. Nieto, J. Phys. A: Math. Theor. 40, 7265 (2007).

[24] E. Merzbacher, Quantum Mechanics (Wiley, New York, 1997).

[25] R. Dick, Advanced Quantum Mechanics - Materials and Photons (Springer - Verlag, New York, 2012).

[26] A.A. Raduta, Nuclear Structure with Coherent States (Springer International Publishing, Cham, 2015).

[27] R. Borrelli e M.F. Gelin, Chem. Phys. 481, 91 (2016).

[28] H.O. Batael, J.F. Silva, A.N. Silva, S.F.M. Santos e E. Drigo Filho, Rev. Bras. Ens. Fís. 40, e2305 (2018).

[29] S. Bittanti, A.J. Laub e J.C. Willems, The Riccati Equation (Springer - Verlag, New York, 2012).

[30] C.V. Sukumar, J. Phys. A: Math. Gen. 18, 2917 (1985).

[31] A.B. Balantekin, Phys. Rev. A 57, 4188 (1998).

[32] E. Drigo Filho e M.A. Cândido, Physica Scripta 64, 548 (2001).

[33] T. Fukui e N. Aizawa, Phys. Lett. A 180, 308 (1993).

[34] S. Gasiorowicz, Quantum Physics (John Wiley \& Sons, New York, 1995), $2^{\mathrm{a}}$ ed.

[35] M. Boas, Mathematical Methods in the Physical Sciences (Wiley, New York, 1983), $2^{\mathrm{a}}$ ed.

[36] G.B. Freitas, R.G. Veigas e E. Drigo Filho, Rev. Bras. Ens. Fís. 32, 1502 (2010).

[37] E. Butkov, Física Matemática (Guanabara Dois, Rio de Janeiro, 1978).

[38] E. Kreyszig, Advanced Engineering Mathematics (Wiley, New York, 1999), $8^{\mathrm{a}}$ ed.

[39] A.N. Ikot e L.E. Akpabio, App. Phys. Res. 2, 202 (2010).

[40] R. Dutt, A. Khare e U.P. Sukhatme, Am. J. Phys 56, 163 (1988). 